\title{
Nonclassical polarization states
}

\author{
Alfredo Luis* \\ Departamento de Óptica, Facultad de Ciencias Físicas, Universidad Complutense, 28040 Madrid, Spain
}

(Received 12 March 2006; published 6 June 2006)

\begin{abstract}
We study nonclassical polarization states characterized by polarization distributions taking negative values. We consider two different families of polarization distributions that include polarization analogs of the Wigner function. We apply different measures of nonclassical behavior such as the nonclassical depth and the distance of the polarization distribution to its modulus. This approach is applied to relevant quantum field states such as one-photon states, SU(2) coherent states, quadrature coherent states, and twin number states. We examine observables allowing the detection of the negativity of the polarization distributions.
\end{abstract}

DOI: 10.1103/PhysRevA.73.063806

PACS number(s): 42.50.Dv, 03.65.Ca, 42.25.Ja

\section{INTRODUCTION}

The basic quality of the quantum theory is the existence of observable results in contradiction with the predictions of classical physics. When this occurs we say that the system is in a nonclassical state.

In this work we focus on nonclassical polarization states. Polarization is a fundamental constituent of light, both in the quantum and in the classical domains. In the quantum regime this variable has been crucial in order to demonstrate experimentally fundamental properties and applications of the quantum theory such as entanglement, complementarity, quantum cryptography, teleportation, and Bell inequalities [1].

We outline the borderline between classical and quantum polarization states with the help of a phase-space description of the problem in which polarization states are represented by distributions on the Poincaré sphere. In this context, nonclassical polarization states are identified as those having distributions with negative values.

The representation of quantum states by phase-space distributions is not unique. For definiteness, in Sec. II we consider families of distributions related to the Wigner function because of their good properties [2]. More specifically, we consider two different approaches. Polarization distributions can be defined as: (i) Marginal distributions obtained from complex-amplitude distributions by removing the variables irrelevant for the specification of polarization, i.e., the total intensity and a global phase $[3,4]$, or (ii) via directly defined distributions purposely introduced for spherical phase spaces associated with angular momenta [5]. This last approach can be applied to polarization since the Stokes operators satisfy the commutation relations of an angular momentum, the associated phase space being the Poincaré sphere.

In Sec. III we assess the amount of nonclassical behavior by translating to polarization two measures of nonclassical behavior previously introduced for complex-amplitude distributions. The first one is the nonclassical depth, defined in terms of the number of distributions that take negative values for a given state [6]. The second one is the distance between

\footnotetext{
*URL: http://www.ucm.es/info/gioq. Electronic address: alluis@ fis.ucm.es
}

the polarization distribution and its modulus [7]. In Sec. IV we apply these ideas to relevant field states such as onephoton states, SU(2) coherent states, quadrature coherent states, and twin number states. In Sec. V we discuss simple practical procedures to detect the negativity of polarization distributions.

\section{POLARIZATION DISTRIBUTIONS}

The basic polarization variables are the Stokes operators $S_{0}$ and $\mathbf{S}$

$$
\begin{aligned}
& S_{0}=a_{1}^{\dagger} a_{1}+a_{2}^{\dagger} a_{2}, \quad S_{y}=i\left(a_{2}^{\dagger} a_{1}-a_{1}^{\dagger} a_{2}\right), \\
& S_{x}=a_{2}^{\dagger} a_{1}+a_{1}^{\dagger} a_{2}, \quad S_{z}=a_{1}^{\dagger} a_{1}-a_{2}^{\dagger} a_{2},
\end{aligned}
$$

where $a_{1}, a_{2}$ are the complex amplitude operators for two field modes [8]. Their mean values are the classical Stokes parameters $\langle\mathbf{S}\rangle$. These operators are formally equivalent to an angular momentum, $\mathbf{S}=2 \mathbf{j}$, allowing the following equivalence between number states and the eigenstates of $j_{z}$ and $\mathbf{j}^{2}$ :

$$
|j, m\rangle=\left|n_{1}=j+m\right\rangle_{1} \otimes\left|n_{2}=j-m\right\rangle_{2},
$$

where $\left|n_{1}\right\rangle_{1},\left|n_{2}\right\rangle_{2}$ are photon-number states in the corresponding mode.

In classical optics the states of definite polarization are specified by Stokes parameters satisfying $\langle\mathbf{S}\rangle^{2}=\left\langle S_{0}\right\rangle^{2}$, which can be represented as a definite point $\langle\mathbf{S}\rangle /\left\langle S_{0}\right\rangle$ in a unit sphere: the Poincaré sphere. The most general polarization state will be described by a distribution on the Poincaré sphere. This is specially the case in the quantum domain where fluctuations of the Stokes variables are unavoidable because of the lack of commutation of the Stokes operators (2.1) $[3,8]$.

In the quantum case there is no unique correspondence between quantum states and polarization distributions. This is due to the lack of commutation of basic quantum observables so that different ordering procedures lead to different correspondences.

In this work we focus on polarization analogs of the socalled $s$-ordered complex-amplitude distributions that embody the Wigner function. There is some consensus in that the Wigner-Weyl correspondence is the one endowed with the largest list of desirable properties [2]. Originally intro- 
duced for Cartesian variables (such as position and linear momentum or field quadratures) the Wigner function has been later adapted to other variables (such as angle and angular momentum, or phase and number) $[5,9,10]$.

Concerning the polarization analogs of quadrature $s$-ordered distributions we can follow two different approaches.

\section{A. Marginal distributions}

We can derive polarization distributions as marginals for the polarization variables of the complex-amplitude $s$-ordered distributions. This is essentially the procedure followed in classical physics [11], which has been further elaborated for the quantum domain in Ref. [4] leading to

$$
W_{s}(\Omega)=\operatorname{tr}\left[\rho \Delta_{s}(\Omega)\right],
$$

where $\rho$ is the density matrix,

$$
\Delta_{s}(\Omega)=\frac{1}{4 \pi}\left(\frac{s+1}{s-1}\right)^{\left(S_{0}-\mathbf{\Omega} \cdot \mathbf{S}\right) / 2}\left(1+\frac{1}{1-s} S_{0}+\frac{1}{1-s} \boldsymbol{\Omega} \cdot \mathbf{S}\right),
$$

$\boldsymbol{\Omega}$ is a three-dimensional real vector

$$
\boldsymbol{\Omega}=\left(\begin{array}{c}
\sin \theta \cos \phi \\
\sin \theta \sin \phi \\
\cos \theta
\end{array}\right)
$$

and $\Omega=(\theta, \phi)$ represents the points of the Poincare sphere in terms of the polar angle $\theta$ and the azimuthal angle $\phi$. We will refer to $W_{s}(\Omega)$ as marginal distributions. The case $s=-1$ has been already applied to the study of the degree of polarization and polarization correlations in quantum optics [3].

\section{B. SU(2) distributions}

On the other hand, we can consider phase-space representations for angular momentum variables derived from first principles [5]

$$
\mathcal{W}_{s}(\Omega)=\operatorname{tr}\left[\rho \Lambda_{s}(\Omega)\right]
$$

where

$$
\Lambda_{s}(\Omega)=\sum_{n=0}^{\infty} \Lambda_{s}(n, \Omega)
$$

with

$$
\begin{aligned}
\Lambda_{s}(n, \Omega)= & \frac{1}{\sqrt{4 \pi}} \sum_{\ell=0}^{2 j} \sum_{m=-\ell}^{\ell} \sum_{k, q=-j}^{j} \sqrt{2 \ell+1} \frac{\langle j, k ; \ell, m \mid j, q\rangle}{\langle j, j ; \ell, 0 \mid j, j\rangle^{s}} Y_{\ell, m}(\Omega) \\
& \times|j, k\rangle\langle j, q|,
\end{aligned}
$$

where $j=n / 2,\left\langle j_{1}, m_{1} ; j_{2}, m_{2} \mid j, m\right\rangle$ are the Clebsch-Gordan coefficients, and $Y_{\ell, m}(\Omega)$ the spherical harmonics. We will refer to $\mathcal{W}_{s}(\Omega)$ as $\mathrm{SU}(2)$ distributions.

\section{Properties}

With the definitions adopted above, both marginal and $\mathrm{SU}(2)$ distributions are real and normalized

$$
\int d \Omega W_{s}(\Omega)=\int d \Omega \mathcal{W}_{s}(\Omega)=1,
$$

being $d \Omega=\sin \theta d \theta d \phi$. They satisfy suitable transformation laws under $\mathrm{SU}(2)$ transformations. These are energy conserving transformations which are linear on the complex amplitudes. In our context they produce rotations of the Stokes operators modifying the position and orientation of the polarization distribution on the Poincaré sphere but preserving their form. They are produced by passive optical devices such as free propagation, beam splitters, phase plates, and mirrors $[3,4,12]$.

Polarization distributions allow us to compute statistical properties by performing phase-space averages as in classical statistical physics. For example, the mean value of a given polarization observable $A(\Omega)$ is

$$
\int d \Omega A(\Omega) W_{s}(\Omega)=\left\langle A_{s}\right\rangle, \quad \int d \Omega A(\Omega) \mathcal{W}_{s}(\Omega)=\left\langle\mathcal{A}_{s}\right\rangle,
$$

where the operators $A_{s}, \mathcal{A}_{s}$ corresponding to the function $A(\Omega)$ are

$$
A_{s}=\int d \Omega A(\Omega) \Delta_{s}(\Omega), \quad \mathcal{A}_{s}=\int d \Omega A(\Omega) \Lambda_{s}(\Omega) .
$$

The converse task, i.e., the determination of the functions corresponding to a given operator $A$, is slightly more involved. Since $\left[\mathbf{S}, S_{0}\right]=\mathbf{0}$ polarization properties must be represented by operators $A$ commuting with the total photon number $S_{0}$. In such a case we can associate with each $A$ a series of functions $A_{s}(n, \Omega), \mathcal{A}_{s}(n, \Omega)$, so that mean values are computed in the form

$$
\begin{aligned}
\langle A\rangle & =\sum_{n=0}^{\infty} \int d \Omega A_{-s}(n, \Omega) W_{s}(n, \Omega) \\
& =\sum_{n=0}^{\infty} \frac{4 \pi}{n+1} \int d \Omega \mathcal{A}_{-s}(n, \Omega) \mathcal{W}_{s}(n, \Omega),
\end{aligned}
$$

where

$$
W_{s}(n, \Omega)=\operatorname{tr}\left[\rho \Delta_{s}(n, \Omega)\right], \quad \mathcal{W}_{s}(n, \Omega)=\operatorname{tr}\left[\rho \Lambda_{s}(n, \Omega)\right],
$$

$$
A_{s}(n, \Omega)=\operatorname{tr}\left[A \widetilde{\Delta}_{s}(n, \Omega)\right], \quad \mathcal{A}_{s}(n, \Omega)=\operatorname{tr}\left[A \Lambda_{s}(n, \Omega)\right],
$$

and $\Delta_{s}(n, \Omega)$ are the restrictions of Eq. (2.4) to the subspace of fixed total photon number $n$, while $\widetilde{\Delta}_{s}(n, \Omega)$ refers to the dual of $\Delta_{s}(n, \Omega)$ introduced in Ref. [4].

By construction, these two families of distributions include suitable analogs of the Wigner function $(s=0)$, the $P$ function $(s=1)$, and the $Q$ function $(s=-1)$.

Marginal and $\mathrm{SU}(2)$ distributions only coincide for $s=$ -1 ( $Q$ function) 


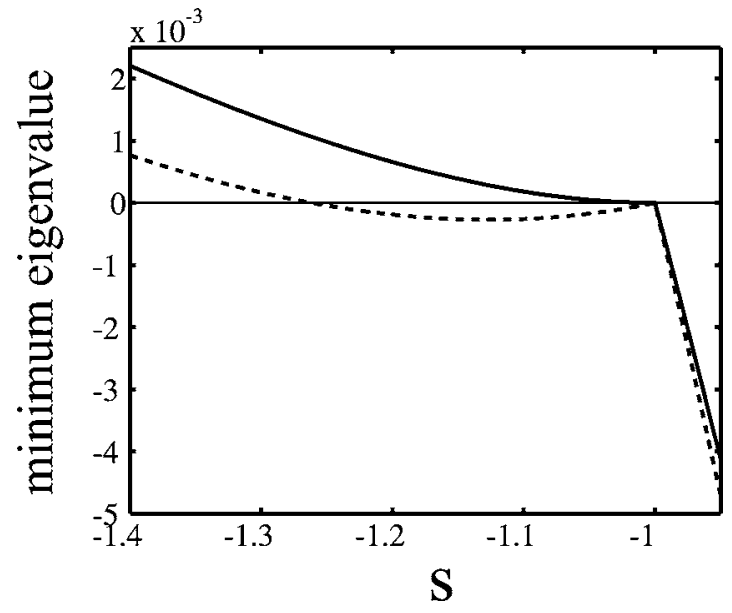

FIG. 1. Minimum eigenvalue of $\Delta_{s}(n, \Omega)$ (solid) and $\Lambda_{s}(n, \Omega)$ (dashed) as functions of $s$ for $j=n / 2=1$.

$$
W_{-1}(\Omega)=\mathcal{W}_{-1}(\Omega)=\sum_{n=0}^{\infty} \frac{n+1}{4 \pi}\langle n, \Omega|\rho| n, \Omega\rangle,
$$

where $|n, \Omega\rangle$ are the $\mathrm{SU}(2)$ coherent states [13]

$$
|n, \Omega\rangle=\sum_{m=0}^{n}\left(\begin{array}{l}
n \\
m
\end{array}\right)^{1 / 2}\left(\sin \frac{\theta}{2}\right)^{n-m}\left(\cos \frac{\theta}{2}\right)^{m} e^{-i m \phi}|m\rangle_{1}|n-m\rangle_{2} .
$$

For $s>-1$ we get that $W_{s}(\Omega)$ and $\mathcal{W}_{s}(\Omega)$ become more and more different as $s \rightarrow 1$, as suitably illustrated by the examples considered in the next section.

It can be appreciated in Eq. (2.15) that the $Q$ function is always positive for every state $W_{-1}(\Omega)=\mathcal{W}_{-1}(\Omega) \geqslant 0$. The universal positivity holds also when $s \leqslant-1$ for the marginal distribution. More explicitly, the eigenvalues of $\Delta(n, \Omega)$ are [4]

$$
\lambda_{s}(j, m)=\frac{1}{4 \pi}\left(1+2 \frac{j+m}{1-s}\right)\left(\frac{s+1}{s-1}\right)^{j-m},
$$

being $j=n / 2$ and $m=-j,-j+1, \ldots, j$, which are all nonnegative $\lambda_{s}(j, m) \geqslant 0$ for $s \leqslant-1$. For the $\mathrm{SU}(2)$ distributions the eigenvalues of $\Lambda_{s}(n, \Omega)$ are [4]

$$
\lambda_{s}(j, m)=\sum_{\ell=0}^{2 j} \frac{2 \ell+1}{4 \pi} \frac{\langle j, m ; \ell, 0 \mid j, m\rangle}{\langle j, j ; \ell, 0 \mid j, j\rangle^{s}} .
$$

In this case $\lambda_{s}(j, m)$ can be negative for $s<-1$, as illustrated in Fig. 1, where we have represented the minimum eigenvalue of both $\Delta_{s}(n, \Omega), \Lambda_{s}(n, \Omega)$ as functions of $s$ for $j$ $=n / 2=1$. Nevertheless, we can appreciate in Fig. 1 that the negativity of $\lambda_{s}(j, m)$ in Eq. (2.18) is very small. Because of this we will consider $s=-1$ as an effective lower bound of $s$ for the analysis of the negativity of polarization distributions. The upper bound for $s$ is discussed in the next section.

\section{NONCLASSICAL DEPTH}

The only nonclassical feature of polarization distributions is that they can take negative values or become more singu- lar than a delta function. Accordingly, polarization states can be considered as nonclassical when $W_{s}(\Omega)<0$ for some $\Omega$ and $s$.

The amount of nonclassical character can be assessed by the number of distributions that take negative values for the same state, the so-called nonclassical depth [6] (other approaches can be found in Refs. [14,15]). More specifically, we can take as a potential measure of nonclassical behavior the quantity $\tilde{s}$ defined as the value of $s$ that separates the regions of positive $(s \leqslant \widetilde{s})$ and negative $(s>\widetilde{s})$ distributions

$$
W_{s \leqslant s}(\Omega) \geqslant 0, \quad W_{s>\hat{s}}(\Omega)<0,
$$

with $s \geqslant-1$. Accordingly, the states with $\tilde{s}=-1$ are the states with the largest nonclassical depth. An equivalent parameter can be defined via $\mathcal{W}_{s}$, leading in general to a different $\tilde{s}$.

It is convenient to restrict from above the range of values of $s$. Otherwise, we might come to the conclusion that every state is nonclassical. We can illustrate this idea with a simple example for quadrature distributions. For every $\rho$ there are always definite values for $s$ and $a$ such that

$$
\int d x d y W_{s}(x, y)\left(x^{2}+\frac{s-a}{2}\right)=\left\langle X^{2}\right\rangle-\frac{a}{2},
$$

where $X$ is the quadrature operator (and thus independent of $s$ ) associated with the function $x^{2}+s / 2$. We can always choose $s$ and $a$ such that $s>a>2\left\langle X^{2}\right\rangle$. In such a case $x^{2}$ $+(s-a) / 2$ is a positive function and Eq. (3.2) implies $W_{s}(x, y)<0$. Therefore, if we do not restrict the values of $s$ we might conclude that every state is nonclassical. For $s$-ordered distributions it is usual to restrict the range of variation of $s$ so that if $\tilde{s} \geqslant 1$ then the state is considered as classical [6].

For fixed $s$ we can compare the amount of nonclassical behavior of different states by simple measures combining physical intuition with simplicity of calculation such as [7]

$$
d_{s}=\int d \Omega\left[\left|W_{s}(\Omega)\right|-W_{s}(\Omega)\right]=\int d \Omega\left|W_{s}(\Omega)\right|-1
$$

and similarly for $\mathcal{W}_{s}(\Omega)$.

We can consider also a slight modification of $d_{s}$ intended to measure negativity as the distance between $W_{s}(\Omega)$ and its modulus $\left|W_{s}(\Omega)\right|$

$$
D_{s}=\int d \Omega\left[W_{s}(\Omega)-\left|W_{s}(\Omega)\right|\right]^{2}
$$

and equivalently for $\mathcal{W}_{s}(\Omega)$

This differs from Eq. (3.3) by the square of the function to be integrated. A similar approach has been already used to compute some other optical properties such as visibility, degree of polarization, and polarization correlations $[3,4,16]$.

Furthermore, a suitable combination of $D_{s}$ and $d_{s}$ 


$$
A_{s}=\frac{d_{s}^{2}}{D_{s}}
$$

provides a measure of the effective area of the Poincare sphere occupied by the negative part of $W_{s}(\Omega)$ [17]. We can note that $d_{s}$ and $D_{s}$ depend on the area of the Poincaré sphere where the distribution takes negative values and also on the values that $W_{s}$ and $\mathcal{W}_{s}$ take on these points. Roughly speaking, $d_{s}, D_{s}$ represent a kind of probability of negative values (being clear that any probabilistic interpretation is ruled out precisely by the negativity). On the other hand, $A_{s}$ tends to represent the area where the distribution takes negative values, irrespective of whether such values are large or small.

It is worth noting that by construction all the above measures are invariant under SU(2) transformations, i.e., they take the same values for the states $\rho$ and $U \rho U^{\dagger}$, where $U$ is any $\mathrm{SU}(2)$ transformation.

\section{NONCLASSICAL POLARIZATION STATES}

In this section we examine the negativity of the polarization distributions corresponding to some relevant field states.

\section{A. One-photon states}

The subspace of states with a single photon shared by two field modes is spanned by the product of number states

$$
|1\rangle_{1}|0\rangle_{2}=\left(\begin{array}{l}
1 \\
0
\end{array}\right), \quad|0\rangle_{1}|1\rangle_{2}=\left(\begin{array}{l}
0 \\
1
\end{array}\right) \text {. }
$$

In this basis we get [4]

$$
\Delta_{s}(\Omega)=\frac{1}{4 \pi}\left(1+\frac{2}{1-s} \boldsymbol{\Omega} \cdot \boldsymbol{\sigma}\right)
$$

and

$$
\Lambda_{s}(\Omega)=\frac{1}{4 \pi}\left(1+\sqrt{3^{s+1}} \boldsymbol{\Omega} \cdot \boldsymbol{\sigma}\right),
$$

where $\boldsymbol{\sigma}$ are the three Pauli matrices. Every one-photon density matrix can be expressed as

$$
\rho=\frac{1}{2}(1+\mathbf{r} \cdot \boldsymbol{\sigma})
$$

where $\mathbf{r}$ is a real vector with $|\mathbf{r}| \leqslant 1$ so that the associated distributions are of the form

$$
W_{s}(\Omega)=\frac{1}{4 \pi}\left(1+\frac{2}{1-s} \mathbf{\Omega} \cdot \mathbf{r}\right)
$$

and

$$
\mathcal{W}_{s}(\Omega)=\frac{1}{4 \pi}\left(1+\sqrt{3^{s+1}} \mathbf{\Omega} \cdot \mathbf{r}\right)
$$

They take negative values provided that $\eta_{s}>1$, where

$$
\eta_{s}=\frac{2|\mathbf{r}|}{1-s}, \quad \eta_{s}=|\mathbf{r}| \sqrt{3^{s+1}}
$$

for the marginal and SU(2) distributions, respectively, leading to

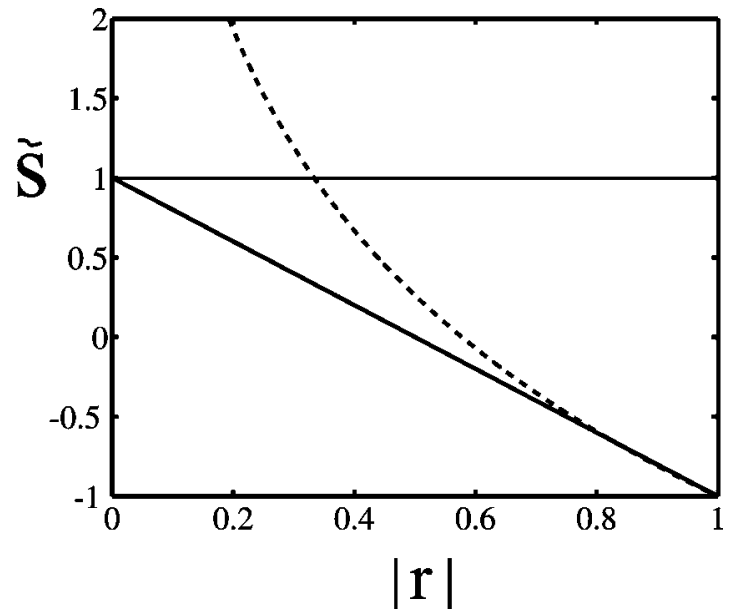

FIG. 2. The $\tilde{s}$ for one-photon states (4.4) as a function of $|\mathbf{r}|$ for marginal (solid) and $\mathrm{SU}(2)$ (dashed) distributions.

$$
\tilde{s}=1-2|\mathbf{r}|, \quad \tilde{s}=-\frac{\ln \left(3|\mathbf{r}|^{2}\right)}{\ln 3},
$$

respectively. This result is illustrated in Fig. 2 .

Note that when considering marginal distributions $W_{s}(\Omega)$ all states are nonclassical polarization states, with the exception of identity $\mathbf{r}=0$, since $\tilde{s}<1$ for all $\mathbf{r} \neq 0$. On the other hand, when considering $\mathrm{SU}(2)$ distributions $\mathcal{W}_{s}(\Omega)$ all the states with $|\mathbf{r}| \leqslant 1 / 3$ would be classical.

In particular, for pure states $|\mathbf{r}|=1$ we get $\tilde{s}=-1$ both for the marginal and the $\mathrm{SU}(2)$ distributions, so that all onephoton pure states have maximal nonclassical polarization distribution.

Concerning $d_{s}, D_{s}$, we get

$$
d_{s}=2 \sinh ^{2}\left(\frac{1}{2} \ln \eta_{s}\right), \quad D_{s}=\frac{\left(\eta_{s}-1\right)^{3}}{6 \pi \eta_{s}} .
$$

In Fig. 3 we have represented $D_{s=0}$ for the marginal and the $\mathrm{SU}(2)$ distributions as functions of $|\mathbf{r}|$, showing that the negativity increases as $\rho$ tends to be pure.

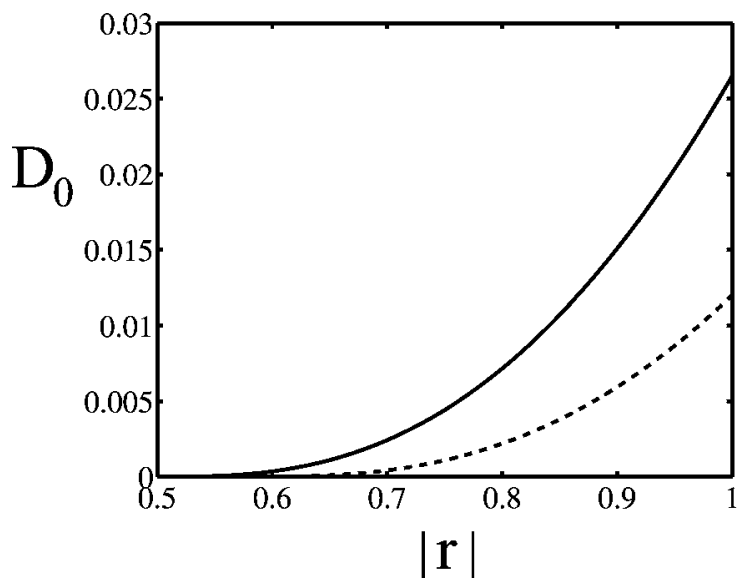

FIG. 3. Plot of $D_{0}$ for marginal (solid) and SU(2) (dashed) distributions as a function of $|\mathbf{r}|$ for one-photon states. 


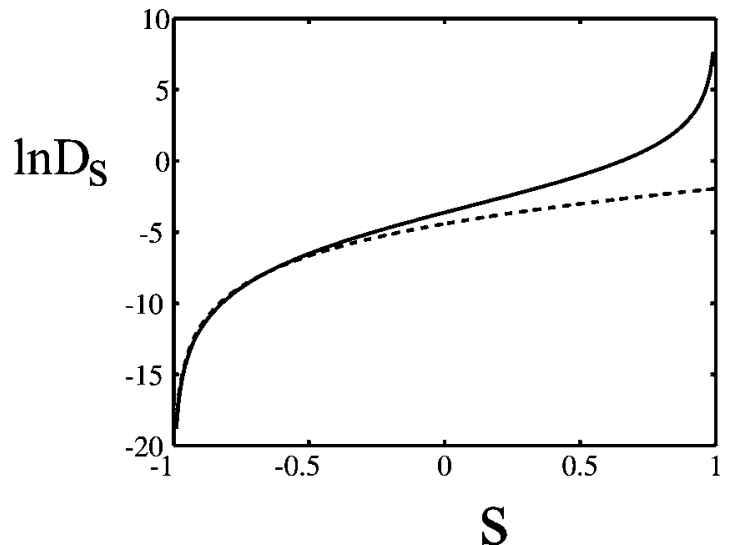

FIG. 4. Plot of $\ln D_{s}$ as a function of $s$ for marginal (solid) and $\mathrm{SU}(2)$ (dashed) distributions for pure one-photon states $|\mathbf{r}|=1$.

In Fig. 4 we have represented $\ln D_{s}$ as a function of $s$ for pure states $|\mathbf{r}|=1$. We can appreciate that the negativity increases as $s \rightarrow 1$. This is a general behavior satisfied by all the examples considered in this work.

We have discussed above that $W_{s}(\Omega), \mathcal{W}_{s}(\Omega)$ coincide for $s=-1$, while their differences tend to increase as $s \rightarrow 1$. We can measure the difference between $W_{s}(\Omega)$ and $\mathcal{W}_{s}(\Omega)$ for the same state by computing the distance

$$
\delta_{s}=\int d \Omega\left[W_{s}(\Omega)-\mathcal{W}_{s}(\Omega)\right]^{2}
$$

that for one-photon states becomes

$$
\delta_{s}=\frac{|\mathbf{r}|^{2}}{12 \pi}\left(\frac{2}{1-s}-\sqrt{3^{1+s}}\right)^{2},
$$

which is represented in Fig. 5 for pure states $|\mathbf{r}|=1$. The difference between $W_{s}(\Omega)$ and $\mathcal{W}_{s}(\Omega)$ increases as $s$ increases. This is a general behavior satisfied by all the examples considered in this work.

Finally, for this simple example we can compute the exact area $\Sigma_{s}$ of the Poincaré sphere where the distributions $W_{s}(\Omega), \mathcal{W}_{s}(\Omega)$ take negative values

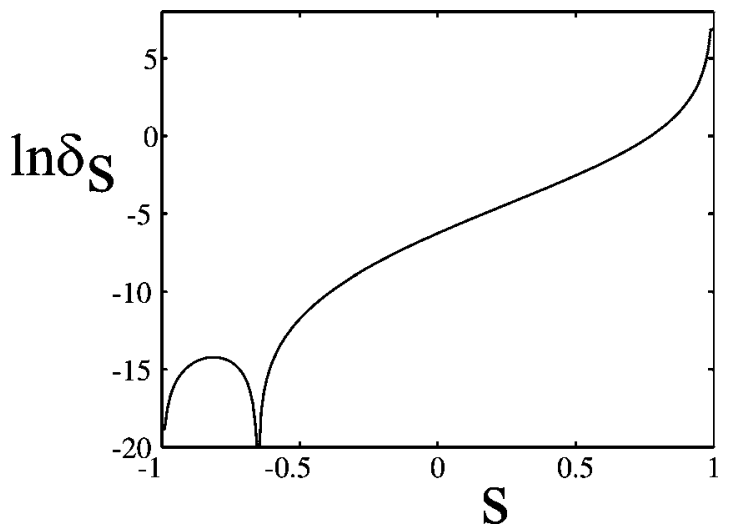

FIG. 5. Plot of the logarithm of the distance $\delta_{s}$ between $W_{s}(\Omega)$ and $\mathcal{W}_{s}(\Omega)$ for pure one-photon states $|\mathbf{r}|=1$.

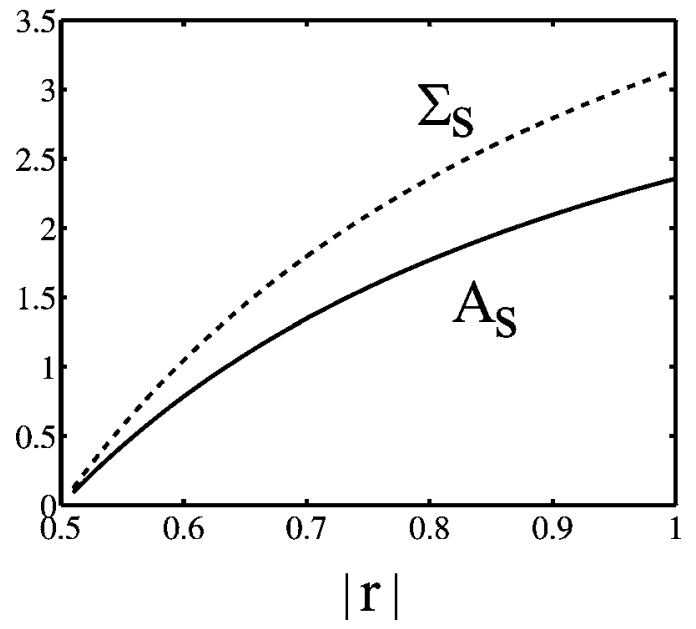

FIG. 6. Plot of $A_{s}$ (solid) and $\Sigma_{s}$ (dashed) as functions of $|\mathbf{r}|$ for the marginal distribution of one-photon states. The unit for $A_{s}$ and $\Sigma_{s}$ is steradian.

$$
\Sigma_{s}=2 \pi\left(1-\frac{1}{\eta_{s}}\right)
$$

In Fig. 6 we have represented both $A_{s}$ and $\Sigma_{s}$ for the $\mathrm{SU}(2)$ distributions as functions of $|\mathbf{r}|$.

\section{B. SU(2) coherent states}

Since all SU(2) coherent states are related by SU(2) transformations we can use the $\mathrm{SU}(2)$ invariance of the formalism considering without loss of generality the simplest case $|n\rangle_{1}|0\rangle_{2}$. The corresponding distributions are [4]

$$
\begin{aligned}
& W_{s}(n, \Omega)=\frac{1}{4 \pi(s-1)^{n}}(s-\cos \theta)^{n-1}[s-n-(n+1) \cos \theta], \\
& \mathcal{W}_{s}(n, \Omega)=\frac{1}{4 \pi} \sum_{\ell=0}^{n}(2 \ell+1)\left\langle\frac{n}{2}, \frac{n}{2} ; \ell, 0 \mid \frac{n}{2}, \frac{n}{2}\right\rangle{ }^{(1-s)} P_{\ell}(\cos \theta),
\end{aligned}
$$

where $P_{\ell}$ are the Legendre polynomials. In Fig. 7 we have represented the Wigner functions $W_{s=0}(\Omega)$ and $\mathcal{W}_{s=0}(\Omega)$ for total photon numbers $n=1,2,3$ as functions of the polar angle $\theta$.

For the marginal distributions $W_{s}(\Omega)$ we get $\tilde{s}=-1$ for all $n \neq 0$, since for $1>s>-1$ the two factors in the first equation in Eq. (4.13) change their sign at different values of $\theta$. This implies that all the SU(2) coherent states would have maximal nonclassical depth.

Concerning the $\mathrm{SU}(2)$ distributions $\mathcal{W}_{s}(\Omega)$, for the lower values of $n$ we have always obtained $\tilde{s}=-1$ for all $n \neq 0$ with the exception of the cases listed in Table I.

In spite of this universal tendency to maximum nonclassical depth, Fig. 7 suggests that the negativity decreases as $n$ increases. This is clearly confirmed by the plot of $D_{0}$ as a function of $n$ in Fig. 8, where it can be appreciated that $D_{0}$ $\rightarrow 0$ as $n$ increases. Similar behavior is observed for $d_{s}$, both for marginal and $\mathrm{SU}(2)$ distributions. This is also the case of 

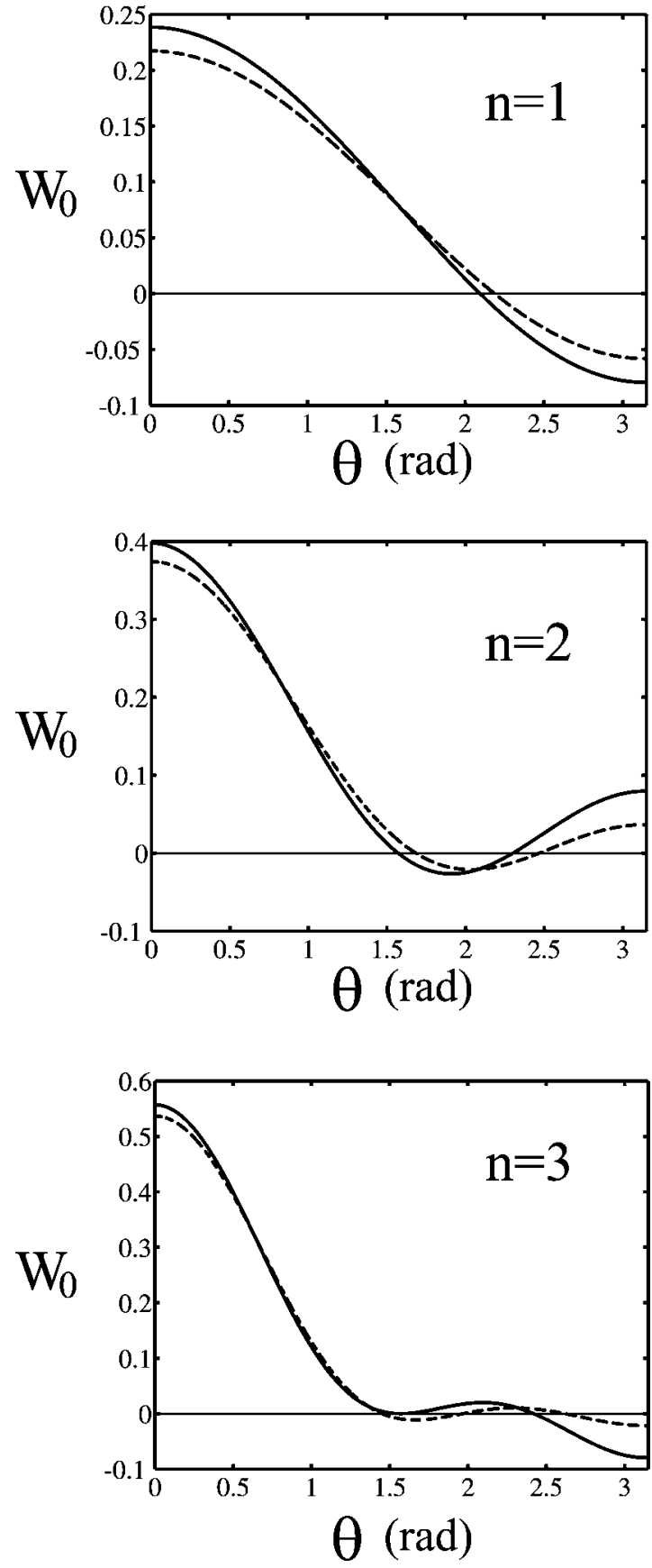

FIG. 7. Marginal (solid) and SU(2) (dashed) Wigner distributions for $\mathrm{SU}(2)$ coherent states at the north pole $|n\rangle_{1}|0\rangle_{2}$ and total photon numbers $n=1,2,3$.

$A_{s}$ for marginal distributions, while $A_{s}$ for $\mathrm{SU}(2)$ distributions shows no clear trend.

On the other hand, it can be also appreciated in Fig. 7 that $W_{0}(\Omega)$ and $\mathcal{W}_{0}(\Omega)$ tend to coincide as $n$ increases. This is confirmed quantitatively by the computation of the distance $\delta_{s}$ in Eq. (4.10), which is represented in Fig. 9 as a function of $n$.

At first sight, the nonclassical polarization properties of SU(2) coherent states might be regarded as somewhat paradoxical, since coherent states are often considered as examples of classical behavior. Nevertheless, there is a strong
TABLE I. Values of $\widetilde{s}$ for SU(2) distributions of SU(2) coherent states with even $n$

\begin{tabular}{lc}
\hline \hline$n$ & $\tilde{s}$ \\
\hline 2 & -0.7826 \\
4 & -0.9673 \\
6 & -0.9998 \\
\hline \hline
\end{tabular}

reason supporting their nonclassical character: For every $\mathrm{SU}(2)$ coherent state there is another $\mathrm{SU}(2)$ coherent state orthogonal to it (centered at antipodal points of the Poincaré sphere). Using the SU(2) Wigner function their orthogonality is expressed as

$$
\int d \Omega \mathcal{W}_{0}(\Omega) \mathcal{W}_{0}^{\prime}(\Omega)=0,
$$

where $\mathcal{W}_{0}(\Omega), \mathcal{W}_{0}^{\prime}(\Omega)$ are the corresponding Wigner functions. Since the SU(2) coherent states are all connected by $\mathrm{SU}(2)$ transformations we have that $\mathcal{W}_{0}(\Omega)$ and $\mathcal{W}_{0}^{\prime}(\Omega)$ are the same function centered at two different points. Therefore, the orthogonality implies that either $\mathcal{W}_{0}(\Omega), \mathcal{W}_{0}^{\prime}(\Omega)$ are disjoint or, otherwise, they must take negative values. For low $n$ the overlap and negativity are large, while as $n$ increases the overlap decreases. This is consistent with the fact that the negativity decreases when $n$ increases, as illustrated in Fig. 8.

\section{Quadrature coherent states}

Taking into account the SU(2) invariance we can compute without loss of generality the polarization distributions for the two-mode quadrature coherent states $|\alpha\rangle_{1}|0\rangle_{2}$ where mode $a_{2}$ is in the vacuum state. Since quadrature coherent states are Poissonian superpositions of SU(2) coherent states $[3,18]$ we get

$$
W_{s}(\Omega)=e^{-\bar{n}} \sum_{n=0}^{\infty} \frac{\bar{n}^{n}}{n !} W_{s}(n, \Omega), \quad \mathcal{W}_{s}(\Omega)=e^{-\bar{n}} \sum_{n=0}^{\infty} \frac{\bar{n}^{n}}{n !} \mathcal{W}_{s}(n, \Omega),
$$

where $\bar{n}=|\alpha|^{2}$ represents the mean number of photons and $W_{s}(n, \Omega)$ and $\mathcal{W}_{s}(n, \Omega)$ are the distributions (4.13) corresponding to $\mathrm{SU}(2)$ coherent states.

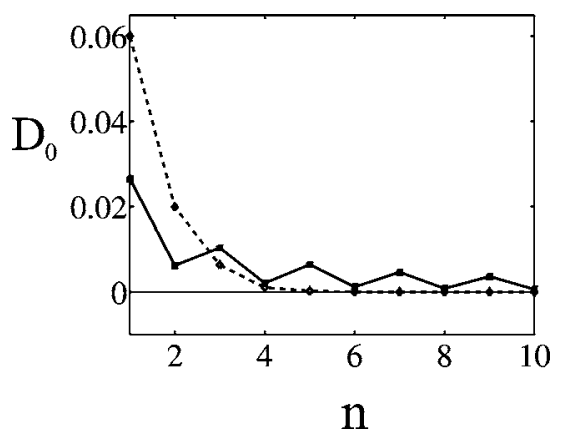

FIG. 8. Plot of $D_{0}$ for the marginal (solid) and $5 D_{0}$ for the SU(2) (dashed) distributions for $\mathrm{SU}(2)$ coherent states as functions of $n$. 


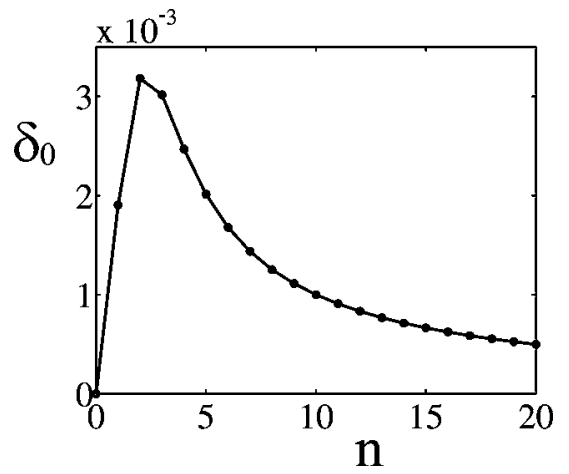

FIG. 9. Plot of the distance $\delta_{s}$ in Eq. (4.10) between the Wigner functions $W_{0}(\Omega)$ and $\mathcal{W}_{0}(\Omega)$ for $\mathrm{SU}(2)$ coherent states as a function of $n$.

For the marginal distribution it is possible to carry out the above sum, leading to

$$
W_{s}(\Omega)=\frac{1}{4 \pi}\left(1+\frac{2 \bar{n}}{1-s} \cos ^{2} \frac{\theta}{2}\right) e^{-2 \bar{n} \sin ^{2}(\theta / 2) /(1-s)},
$$

which is always non-negative for all $1>s \geqslant-1$.

In Fig. 10 we have represented the marginal and SU(2) Wigner functions for a coherent state of mean number of photons $\bar{n}=4$. The marginal and $\mathrm{SU}(2)$ distributions are very close and can hardly be distinguished. Nevertheless, there is a significant difference, since the $\mathrm{SU}(2)$ Wigner function takes negative values around $\theta=\pi$ as illustrated by the inset in Fig. 10 [19].

The values of $\widetilde{s}$ for the $\mathrm{SU}(2)$ distributions are shown in Table II for $\bar{n} \leqslant 20$. They suggest that the nonclassical depth increases as $\bar{n}$ increases. However, the evaluation of $D_{0}$ suggests that the nonclassical polarization behavior occurs exclusively for small values of $\bar{n}$. This is illustrated in Fig. 11 where we have represented $D_{0}$ for the $\mathrm{SU}(2)$ Wigner function

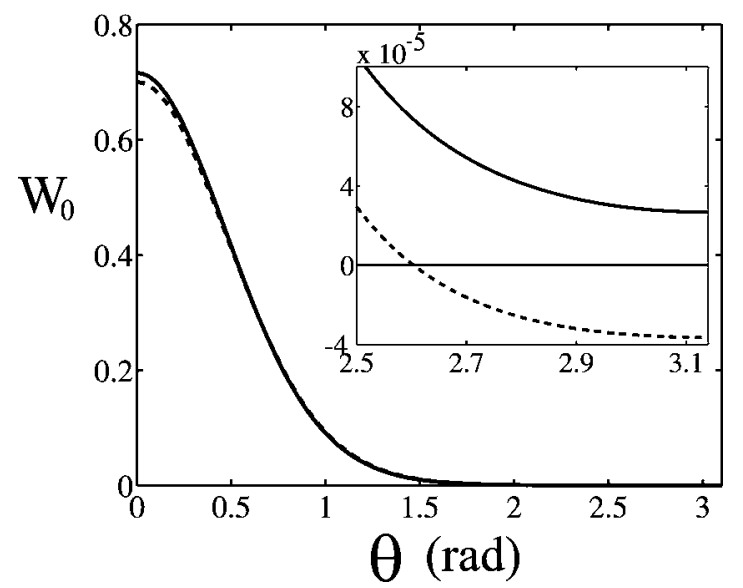

FIG. 10. Marginal (solid) and SU(2) (dashed) Wigner distributions for a quadrature coherent state centered at the north pole $|\alpha\rangle_{1}|0\rangle_{2}$ with $\bar{n}=4$. The inset shows in detail the area around the south pole where the $\mathrm{SU}(2)$ distribution takes negative values while the marginal remains positive.
TABLE II. Values of $\widetilde{s}$ for quadrature coherent states for several values of $\bar{n}$

\begin{tabular}{lc}
\hline \hline $\bar{n}$ & $\tilde{s}$ \\
\hline 2 & 0.36 \\
4 & -0.07 \\
6 & -0.24 \\
8 & -0.33 \\
10 & -0.38 \\
12 & -0.41 \\
14 & -0.44 \\
16 & -0.46 \\
18 & -0.48 \\
20 & -0.49 \\
\hline \hline
\end{tabular}

as a function of the mean number of photons $\bar{n}$, showing that the largest negativity occurs when $\bar{n} \simeq 5$. A similar dependence on $\bar{n}$ is displayed by $d_{0}$, while $A_{0}$ shows no clear trend.

This result is relevant since quadrature coherent states are often considered as examples of classical behavior. We can recall that there are other phase-space formalisms that assign negative distributions to quadrature coherent states [10,20].

\section{Twin number states $|n\rangle_{1}|n\rangle_{2}$}

Another interesting class of field states is the twin number states $|n\rangle_{1}|n\rangle_{2}$ which are closely related to nonclassical features such as polarization squeezing [8]. In Fig. 12 we have represented the Wigner functions for the states with $n$ $=1,2,3$, (all of them independent of the azimuthal angle $\phi$ ) as functions of the polar angle $\theta$.

For the marginal distributions $W_{s}$ we have obtained $\widetilde{s}=$ -1 for all the cases examined. Concerning the SU(2) distributions $\mathcal{W}_{s}$, for low values of $n$ we have obtained $\tilde{s}=-1$ with the exception of $n=2,4$, for which $\widetilde{s}=-0.87,-0.99$, respectively.

Nevertheless, although the nonclassical depth $\widetilde{s}$ is maximal for most $n$, the evaluation of $D_{s}$ suggests that the nonclassical character increases as $n$ increases, as illustrated in Fig. 13. This is in sharp contrast to the behavior of $\mathrm{SU}(2)$

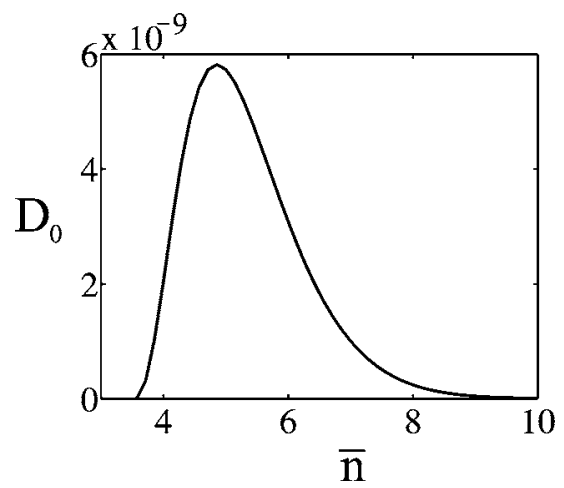

FIG. 11. Plot of $D_{0}$ for the SU(2) polarization distribution of quadrature coherent states as a function of the mean number of photons $\bar{n}$. 

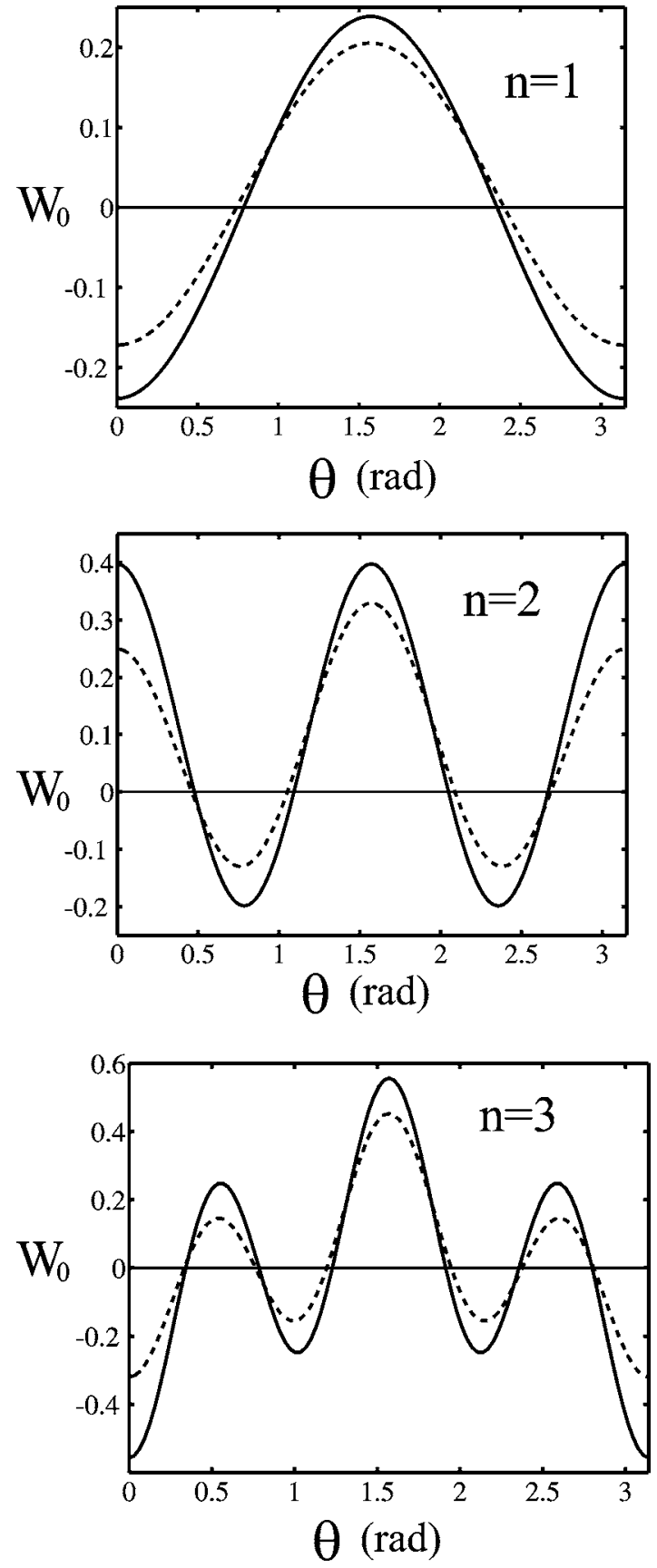

FIG. 12. Marginal (solid) and SU(2) (dashed) Wigner distributions for the states $|n\rangle_{1}|n\rangle_{2}$ for $n=1,2,3$.

coherent states, whose negativity decreases as $n$ increases.

Furthermore, for the states $|n\rangle_{1}|n\rangle_{2}$ the difference between $W_{0}(\Omega)$ and $\mathcal{W}_{0}(\Omega)$ increases as $n$ increases, as illustrated in Fig. 14. This is also in sharp contrast to the behavior of SU(2) coherent states for which $W_{0}(\Omega)$ and $\mathcal{W}_{0}(\Omega)$ tend to coincide as $n$ increases.

\section{OBSERVATION OF NEGATIVITY}

In practical terms, the nonclassical polarization properties implied by negative distributions should be revealed by measurement outputs outside the classical range of variation for

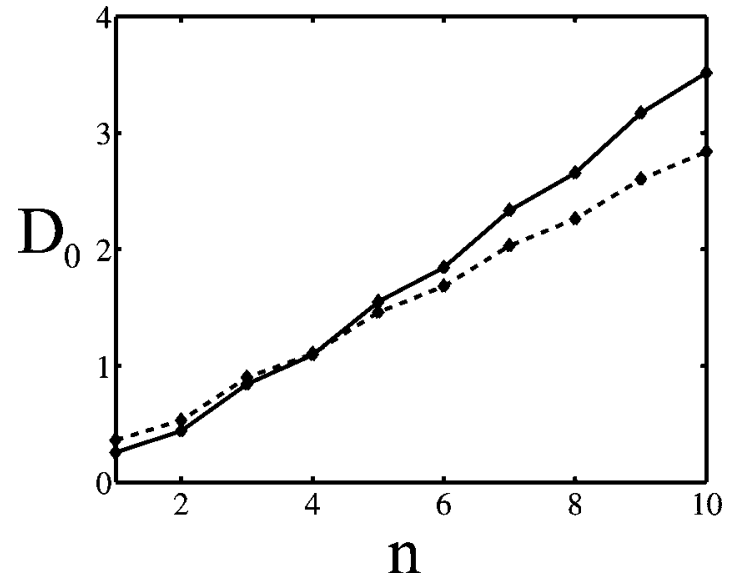

FIG. 13. Plot of $D_{0}$ for the marginal distribution (solid) and $3 D_{0}$ for the $\mathrm{SU}(2)$ distribution (dashed) for the states $|n\rangle_{1}|n\rangle_{2}$ as functions of $n$.

the measured observable (such as negative mean values for observables with positive phase-space representatives). In this section we examine whether there are observables $A$ with $A(\Omega) \geqslant 0$ and $\langle A\rangle<0$.

The answer is affirmative since we can always choose observables with a positive phase-space representative centered at the region where $W_{s}(\Omega)<0$ and with widths as narrow as desired. For example, for states taking negative values at the south pole of the Poincaré sphere, a suitable observable revealing the negativity is

$$
A(\Omega)=\frac{k+1}{4 \pi} \sin ^{2 k} \frac{\theta}{2},
$$

which is always positive, taking meaningful values exclusively on a region around the south pole that becomes smaller as $k$ increases.

For one-photon states we have from (2.11), (4.2), and (4.3) that the restriction to the one-photon subspace of the quantum operators corresponding to $A(\Omega)$ is

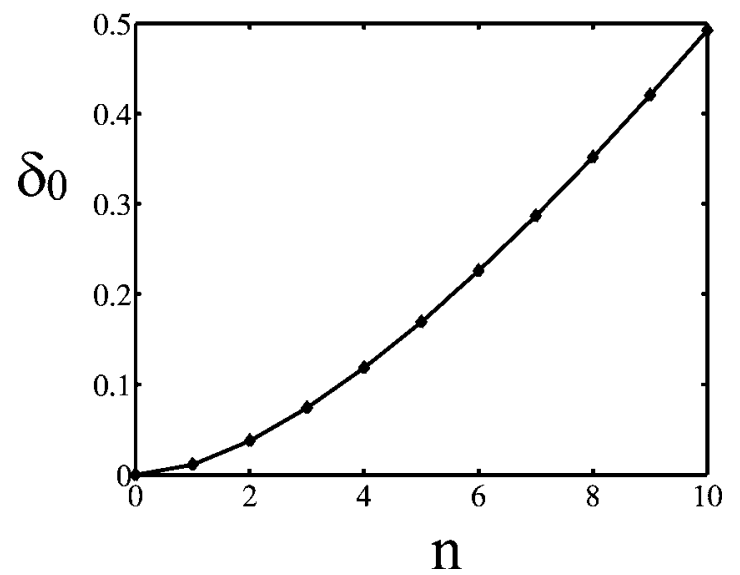

FIG. 14. Plot of the distance $\delta_{0}$ in Eq. (4.10) between $W_{0}(\Omega)$ and $\mathcal{W}_{0}(\Omega)$ for the states $|n\rangle_{1}|n\rangle_{2}$ as a function of $n$. 


$$
\begin{aligned}
& A_{s}=\frac{1}{4 \pi}\left(1-\frac{2}{1-s} \frac{k}{k+2} S_{z}\right), \\
& \mathcal{A}_{s}=\frac{1}{4 \pi}\left(1-\sqrt{3^{s+1}} \frac{k}{k+2} S_{z}\right),
\end{aligned}
$$

for marginal and SU(2) distributions, respectively. Therefore, the negativity of $W_{s}$ and $\mathcal{W}_{s}$ can be easily revealed by photon-number measurements. For example, for the state $|1\rangle_{1}|0\rangle_{2}$ we get evidence of negative character of the polarization distributions provided that

$$
k>2 \frac{1-s}{1+s}, \quad k>\frac{2}{\sqrt{3^{s+1}}-1}
$$

for the marginal and the SU(2) distributions, respectively. A similar approach can be considered for $\mathrm{SU}(2)$ and quadrature coherent states. Further details can be found in Ref. [19].

As a further example we consider the state $|1\rangle_{1}|1\rangle_{2}$. In this case the negativity of the Wigner distributions $s=0$ can be disclosed by a negative mean value for an observable with positive distribution such as $A(\Omega)=\cos ^{2} \theta$. The restriction of the corresponding operators with $s=0$ to the two-photon subspace is

$$
\begin{gathered}
A_{0}=\frac{1}{5}\left(S_{z}^{2}-1\right), \\
\mathcal{A}_{0}=\frac{1}{12 \sqrt{10}} S_{z}^{2}+\frac{\sqrt{10}-4}{3 \sqrt{10}},
\end{gathered}
$$

for the marginal and the SU(2) distributions, respectively, with

$$
\left\langle A_{0}\right\rangle=-\frac{1}{5}, \quad\left\langle\mathcal{A}_{0}\right\rangle=\frac{\sqrt{10}-4}{3 \sqrt{10}} \simeq-0.0883,
$$

respectively.

\section{CONCLUSIONS}

Quantum systems with nonpositive polarization distributions can be identified as nonclassical polarization states. To this end we have examined different polarization distributions and different measures of the amount of negativity. In this regard, it seems that the nonclassical depth is rather insensible and tends to its maximum value very often. Because of this we have completed it by computing some other intuitive and simple measures of negativity in terms of the distance between the polarization distribution and its modulus.

We have found that $\mathrm{SU}(2)$ coherent states and twin photon number states possess strong nonclassical polarization distributions. While the negativity of the SU(2) coherent states decreases as the number of photons increases the negativity of twin photon number states increases as the number of photons increases.

We have shown that quadrature coherent states display nonclassical polarization properties. In comparison with previous proposals of nonclassical behavior for coherent states, the approach presented in this work is conceptually much more simple and suitable to be experimentally implemented by simple photon number measurements.

\section{ACKNOWLEDGMENT}

A.L. acknowledges the support from Project No. FIS2004-01814 of the Spanish Dirección General de Investigación del Ministerio de Educación y Ciencia.
[1] The Physics of Quantum Information, edited by D. Bouwmeester, A. Ekert, and A. Zeilinger (Springer, Berlin, 2000).

[2] K. E. Cahill and R. J. Glauber, Phys. Rev. 177, 1857 (1969); 177, 1882 (1969); M. Hillery, R. F. O’Connell, M. O. Scully, and E. P. Wigner, Phys. Rep. 106, 121 (1984); B.-G. Englert, J. Phys. A 22, 625 (1989); H.-W. Lee, Phys. Rep. 259, 147 (1995)

[3] A. Luis, Phys. Rev. A 66, 013806 (2002); Opt. Commun. 216, 165 (2003); Phys. Rev. A 69, 023803 (2004).

[4] A. Luis, Phys. Rev. A 71, 053801 (2005).

[5] G. S. Agarwal, Phys. Rev. A 24, 2889 (1981); J. C. Várilly and J. M. Gracia-Bondía, Ann. Phys. (N.Y.) 190, 107 (1989); J.-P. Amiet and M. B. Cibils, J. Phys. A 24, 1515 (1991); G. S. Agarwal, D. Home, and W. Schleich, Phys. Lett. A 170, 359 (1992); G. S. Agarwal, Phys. Rev. A 47, 4608 (1993); J. P. Dowling, G. S. Agarwal, and W. P. Schleich, ibid. 49, 4101 (1994); C. Brif and A. Mann, J. Phys. A 31, L9 (1998); M. P. de Miranda, F. J. Aoiz, L. Bañares, and V. Saéz Rábanos, J. Chem. Phys. 111, 5368 (1999); D. Arsenović, N. Burić, and D. M. Davidović, Phys. Rev. A 60, 1797 (1999); C. Brif and A. Mann, ibid. 59, 971 (1999); J. Opt. B: Quantum Semiclassical
Opt. 2, 245 (2000); A. B. Klimov and S. M. Chumakov, J. Opt. Soc. Am. A 17, 2315 (2000); J. P. Amiet and S. Weigert, Phys. Rev. A 63, 012102 (2001); M. P. de Miranda and F. J. Aoiz, Phys. Rev. Lett. 93, 083201 (2004).

[6] C. T. Lee, Phys. Rev. A 44, R2775 (1991); 52, 3374 (1995); N. Lütkenhaus and S. M. Barnett, ibid. 51, 3340 (1995); A. F. de Lima and B. Baseia, ibid. 54, 4589 (1996); J. Janszky, M. G. Kim, and M. S. Kim, ibid. 53, 502 (1996).

[7] M. G. Benedict and A. Czirják, Phys. Rev. A 60, 4034 (1999); A. Kenfack and K. Zyczkowski, J. Opt. B: Quantum Semiclassical Opt. 6, 396 (2004); e-print quant-ph/0406015.

[8] A. Luis and L. L. Sánchez-Soto, in Progress in Optics, edited by E. Wolf (Elsevier, Amsterdam, 2000), Vol. 41, p. 421, and references therein.

[9] N. Mukunda, Am. J. Phys. 47, 182 (1979); J. P. Bizarro, Phys. Rev. A 49, 3255 (1994); P. Kasperkovitz and M. Peev, Ann. Phys. (N.Y.) 230, 21 (1994); D. M. Kaplan and G. C. Summerfield, Phys. Rev. 187, 639 (1969); M. O. Scully, Phys. Rev. D 28, 2477 (1983); G. Ramachandran, A. R. Usha-Devi, P. Devi, and S. Sirsi, Found. Phys. 26, 401 (1996); S. M. Chumakov, A. Frank, and K. B. Wolf, Phys. Rev. A 60, 1817 
(1999); V. P. Karassiov and A. V. Masalov, J. Opt. B: Quantum Semiclassical Opt. 4, S366 (2002); S. M. Chumakov, A. B. Klimov, and K. B. Wolf, Phys. Rev. A 61, 034101 (2001); W. K. Wootters, Ann. Phys. (N.Y.) 176, 1 (1987); O. Cohendet, $\mathrm{Ph}$. Combe, M. Sirugue, and M. Sirugue-Collin, J. Phys. A 21, 2875 (1988); D. Galetti and A. F. R. Toledo Piza, Physica A 149, 267 (1988); A. Lukš and V. Peřinová, Quantum Opt. 6, 125 (1994); U. Leonhardt, Phys. Rev. Lett. 74, 4101 (1995); Phys. Rev. A 53, 2998 (1996); T. Opatrný, D.-G. Welsch, and V. Bužek, ibid. 53, 3822 (1996); A. Luis and J. Peřina, J. Phys. A 31, 1423 (1998); C. Miquel, J. P. Paz, and M. Saraceno, Phys. Rev. A 65, 062309 (2002); J. P. Paz, ibid. 65, 062311 (2002); K. S. Gibbons, M. J. Hoffman, and W. K. Wootters, ibid. 70, 062101 (2004); S. Chaturvedi, E. Ercolessi, G. Marmo, G. Morandi, N. Mukunda, and R. Simon, J. Phys. A 39, 1405 (2006).

[10] J. Vaccaro, Opt. Commun. 113, 421 (1995); Phys. Rev. A 52, 3474 (1995); J. Vaccaro and D. T. Pegg, ibid. 41, 5156 (1990).

[11] Ch. Brosseau, Fundamentals of Polarized Light: A Statistical Optics Approach (Wiley, New York, 1998).

[12] A. Luis and L. L. Sánchez-Soto, Quantum Semiclassic. Opt. 7, 153 (1995).

[13] F. T. Arecchi, E. Courtens, R. Gilmore, and H. Thomas, Phys. Rev. A 6, 2211 (1972).

[14] M. Hillery, Phys. Rev. A 35, 725 (1987); P. Kazimierczyk and A. Orłowski, Phys. Lett. A 149, 449 (1990); V. V.
Dodonov, O. V. Man'ko, V. I. Man'ko, and A. Wünsche, J. Mod. Opt. 47, 633 (2000); P. Marian, T. A. Marian, and H. Scutaru, Phys. Rev. Lett. 88, 153601 (2002); J. M. C. Malbouisson and B. Baseia, e-print quant-ph/0209107; Phys. Scr. 67, 93 (2003); V. V. Dodonov and M. B. Renó, Phys. Lett. A 308, 249 (2003); P. Marian, T. A. Marian, and H. Scutaru, Phys. Rev. A 69, 022104 (2004); J. K. Asbóth, J. Calsamiglia, and H. Ritsch, Phys. Rev. Lett. 94, 173602 (2005); e-print quant$\mathrm{ph} / 0411164$.

[15] R. R. Puri, J. Phys. A 29, 5719 (1996).

[16] A. Luis, J. Phys. A 35, 8805 (2002); Phys. Lett. A 314, 197 (2003); Phys. Rev. A 71, 063815 (2005).

[17] E. J. Heller, Phys. Rev. A 35, 1360 (1987); H. Maassen and J. B. M. Uffink, Phys. Rev. Lett. 60, 1103 (1988); B. Mirbach and H. J. Korsch, Ann. Phys. (N.Y.) 265, 80 (1998); M. J. W. Hall, Phys. Rev. A 59, 2602 (1999); S. Gnutzmann and K. Życzkowski, J. Phys. A 34, 10123 (2001).

[18] P. W. Atkins and J. C. Dobson, Proc. R. Soc. London, Ser. A 321, 321 (1971); A. Luis and J. Peřina, Phys. Rev. A 53, 1886 (1996).

[19] A. Luis (unpublished).

[20] L. M. Johansen, Phys. Lett. A 329, 184 (2004); J. Opt. B: Quantum Semiclassical Opt. 6, L21 (2004); e-print quant-ph/ 0309025; e-print quant-ph/0402105; L. M. Johansen and A. Luis, Phys. Rev. A 70, 052115 (2004). 